\title{
A FRAMEWORK FOR PREDICTING POTENTIAL PRODUCT IMPACT DURING PRODUCT DESIGN
}

\author{
Mabey, Christopher S.; \\ Armstrong, Andrew G.; \\ Mattson, Christopher A.; \\ Salmon, John L.; \\ Hatch, Nile W. \\ Brigham Young University
}

\begin{abstract}
The impact of products is becoming a topic of concern in society. Product impact may fall under the categories of economic, environmental, or social impact and is defined by the effect of a product on day-to-day life. Design teams lack sufficient tools to predict the impact of products they are designing. In this paper we present a framework for the prediction of product impact during product design. This framework integrates models of the product, scenario, society, and impact into an agent-based model to predict product impact. Although this paper focuses on social impact, the framework can also be applied to economic or environmental impacts. An illustration of using the framework is also presented. Agent-based modeling has been used previously for adoption models, but it has not been extended to predict product impact. Having tools for impact prediction allows for optimizing the product design parameters to increase potential positive impact and reduce potential negative impact.
\end{abstract}

Keywords: Product modelling / models, Social responsibility, Societal consequences

\section{Contact:}

Mabey, Christopher S.

Brigham Young University

Mechanical Engineering

United States of America

csmabey@gmail.com 


\section{INTRODUCTION}

In the twenty-first century the world is seeing an ever increasing call for businesses to do more than maximize shareholder value (Gelles and Yaffe-Bellany, 2019). The rapid rise of the benefit corporation - a company that includes positive impact on society as a goal- demonstrates an increased interest in the impact of products and companies (Chen and Kelly, 2015). Product impact generally falls into three categories: economic, environmental, and social. Emerging sectors such as engineering for global development center their mission around utilizing engineering and design to provide positive impact (Mattson and Winter, 2016). There is increased discussion around product impact but few tools exist to predict it or plan well for it.

Interviews with practicing engineers show that most consider the social impact of the product they design, but that focus is mostly on ensuring that products are safe to use and not on other social impacts such as impacts on education, income, or family (Pack et al., 2019). It has been acknowledged that more tools need to be developed to help designers predict the social impact of products in order to make better design choices (Pack et al., 2019). To predict the social impact of a product, a model needs to have methods for (i) social impact measurement, and (ii) the adoption and diffusion of technology.

In this paper we present a framework that couples existing methods for measuring impact and technology diffusion, and combine them in an agent-based model (ABM). Although the framework may be used to model economic, environmental, and social impact, this paper mainly focuses on social impact. This framework will allow for building ABM to predict social impact as a design tool so that the product design team can make choices to increase the positive impact and reduce the negative impact of the product before it enters the market. The different components of this framework have been used previously in the literature, but they have not been combined into a workflow to predict product impacts. Following the description of the framework, an illustration of how the framework can be used is provided. Tools such as this framework will help design teams make decisions about what aspects of a product are most important to increase potential positive impact while limiting potential negative impact.

\subsection{Social Impact Measurement}

The social impact of a product can be defined as its influence on a person's day-to-day life (Burdge, 2015). All products have social impact which can be positive or negative (Norman and MacDonald, 2004). Literature on measuring social impact has been growing and frameworks for assessing the social impact of products have emerged such as Social Impact Assessment (SIA) (Fontes et al., 2018), the Social Life Cycle Assessment of Products (Benoît et al., 2010), and the Product Impact Metric. These frameworks were largely built on assessing current products and not on predicting social impacts of a future product. There has been emerging work in the area of social impact predictive modeling (Stevenson et al., 2020). In order to make a prediction of social impact one must gain a knowledge of the societies and stakeholders that will be potentially affected (Esteves et al., 2012), decide upon areas of potential social impact (Rainock et al., 2018), what stakeholders to focus on (Fontes et al., 2016), and relevant measures for the given category and stakeholder (Stevenson et al., 2020). These methods are important to design teams to gain an understanding of how a product affects a persons day-to-day life rather than merely focusing on increasing the number of units sold.

\subsection{Adoption and Diffusion of Technology}

Theories on adoption and diffusion of technology can be split into how a technology spreads across society on a macro scale and how a single individual decides whether to adopt a particular technology. The Diffusion of Innovations (Rogers, 1995) with its familiar s-curve of adoption focuses on the diffusion of a technology through society on an aggregate level. Theories such as the theory of planned behavior (TPB) (Ajzen, 1991) and the unified theory of acceptance and use of technology (UTAUT) (Venkatesh et al., 2003) focus on the factors that cause an individual to adopt a technology or behavior. To implement TPB or UTAUT, information must be gathered about individual user preferences to build a model.

This paper focuses on using the TPB, due to being more pragmatic to implement a model from secondary data. Adoption models have utilized TPB to capture the intentions of users to adopt a technology (Pavlou and Fygenson, 2006). TPB has three main components that affect an individual's choice of adopting a behavior: (i) attitude toward the behavior, (ii) social norms, and (iii) perceived behavioral control. Data 
from the TPB can be integrated into a discrete choice model to determine probabilities of a user adopting a behavior (Pakravan and MacCarty, 2020).

\subsection{Agent Based Modeling}

$\mathrm{ABM}$ is a method for describing the behavior of a system by modeling it as a collection of individual entities called agents (Bonabeau, 2002). Each individual agent acts independently and makes decisions based on the agent's definition and model parameters. Much of the value of ABM comes from observing the patterns that emerge as agents interact. This emergent behavior can be unexpected even from a simple model with just a few rules (Axelrod, 1997). ABM differs from more traditional differential modeling in that it does not seek for a top-down governing equation for modeling the system behavior, rather it builds the system from the bottom-up with individual agents (Vicsek, 2002). ABM has been used to model segregation (Schelling, 1971), social behavior (Smaldino et al., 2012), and adoption (Rai and Robinson, 2015), among many other applications (Squazzoni, 2010).

The bottom-up approach of ABM allows for combining social factors into the modeling of adoption using Discrete Choice Analysis (DCA) (He et al., 2014). By building on adoption models, this paper adds measures of impact categories through key indicators for an agent adopting or not adopting a product. The addition of these impact indicators enables ABM to predict product impacts while a product is still in the early design stage.

\section{PRODUCT IMPACT MODELING FRAMEWORK}

In order to model the impact of a product there must be a model for the product, and a model for society. The framework that is presented combines model types in a novel way to extend modeling beyond product adoption - where most models stop - to that of product impact. An overview of the framework can be seen in Figure 1. Models for the product, society, scenario, and impact are created and integrated in an ABM. The ABM will output data that will make up the impact assessment. Product impact data is fed back to the product model to iterate on design parameters to maximize the potential positive impact of the product. Each of these components is explained in detail below.

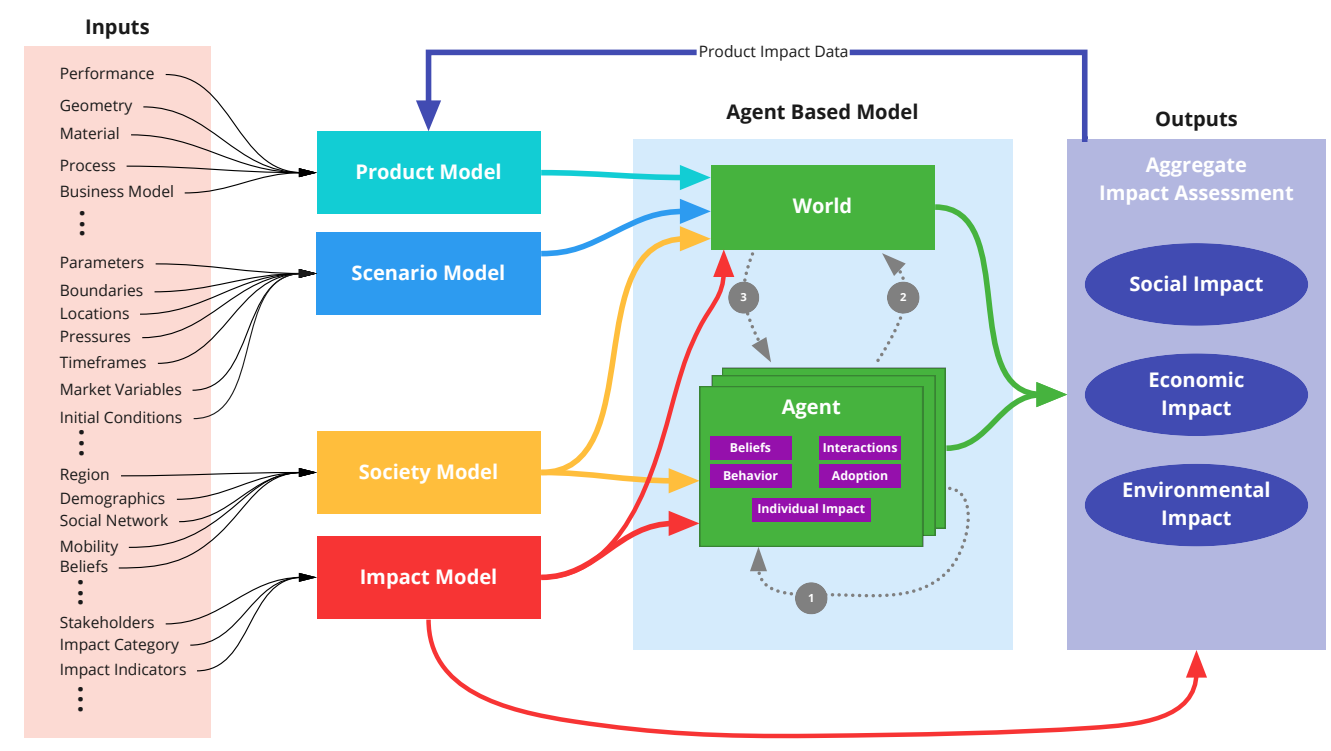

Figure 1. Product impact framework structure

\subsection{Framework: Product Model}

This framework is intended to be used during product development when the design is still somewhat flexible, but enough must be known about the product's design to predict its performance. Items such as the performance, geometry, material, and potential business model may be incorporated into the 
product model. Essential product attributes that may influence the product impact should be chosen to act as inputs for the ABM. For example a designer might choose efficiency, comfort, and ease of use to examine, explore, and iterate over. These attributes will also be used in the DCA as a part of an individual agent's choice of adoption. Adoption modeling using DCA will be covered in depth in Section 2.5 .

\subsection{Framework: Society Model}

The first step in building a society model is selecting a target population for the simulation. There are two types of information needed about a target population: data on user attitudes, behaviors and preferences, and demographic data to build a synthetic population in the model.

Data on user preferences can be collected through surveys and other means from potential users. This survey includes how important the selected product parameters are to a potential user, as well as information related to the TPB categories of attitude, social norms, and perceived behavioral control surrounding the adoption of the product (Ajzen, 1991). This framework focuses on a binary choice model, where the choice is to adopt or not adopt the product (Wassenaar and Chen, 2003). A multinomial choice model may be considered where a user will choose between competing products, but this leads to a more complex model that is outside the scope of this paper due to needing preference data about competing products. Data about user preferences is needed for creating the adoption probability equations that will be discussed in Section 2.5.

The other set of data is about demographics and behavior of the target population. This data is used to inform agent actions and interactions. When complete knowledge of a population is not available, a synthetic population may be used. Synthetic populations create demographic data sets for agents that match the distribution of the population of interest (Müller and Axhausen, 2010). Synthetic populations allow for more accurate simulation of both the individual behavior of agents, and interactions between agents that are affected by individual level demographics (Gargiulo et al., 2010). This helps agent based models capture emergent behavior due to societal behavior (Xu et al., 2017).

Another key structure of the society model is the social network structure of the society. This model utilizes a small-world network topology (Watts and Strogatz, 1998) that has individuals within the network connect to $k$ closest neighbors, and each individual has a probability of rewiring a connection to that of another random individual from the entire population. Small-world networks have been used widely in ABM adoption and diffusion models (Rai and Robinson, 2015; Schwarz and Ernst, 2012) because they have been shown to follow patterns found in real-world social networks (Latora and Marchiori, 2001).

\subsection{Framework: Scenario Model}

The scenario model used in this paper defines the scope of the ABM. This includes model parameters such as the duration of the simulation, physical attributes of the world created for agents, and initial conditions of inputs for the ABM (Macal and North, 2005). Data gathered for the society model helps inform building the physical attributes of the scenario with items such as number of households or workplaces that may need to be created in the modeled world. It should be decided if one or multiple scenarios will be examined as one may be interested in the product impact over different contexts. To ensure comparability, all scenarios should be run through the same simulations of the product parameters.

\subsection{Framework: Impact Model}

Much of the literature on assessing the social impact of products that are on the market will also apply to predicting impact. As outlined in SIA and Social Life Cycle Assessment, categories of impact need to be decided upon and then indicators selected to measure the impact categories (Fontes et al., 2018; Benoit et al., 2010). Work on predictive social impact modeling adds the importance of determining how product parameters link to impact indicators (Stevenson et al., 2020). The framework in this paper utilizes the eleven categories from Rainock et al. (2018) for social impact category selection. The amount of resources required to collect data will increase with the number of selected impact categories and indicators. It is important for the design team to narrow down the focus of the model to the amount of impact categories that will enable data collection with available resources. Data from users should inform the selection of impact categories. 


\subsection{Framework: Agent-Based Model}

Inputs from the product, society, scenario, and impact models are integrated into an ABM. These inputs define the world that agents exist within, and the agents themselves. One of the key characteristics of any $\mathrm{ABM}$ is defining how agents interact with each other and the world (Bonabeau, 2002), these interactions are shown by the dashed arrows labeled 1,2, and 3 in Figure 1. Agents may influence each other, such as when an agent has a poor experience with a product and their attitude towards the product becomes more negative, and then influences attitudes of other agents. The world may influence agents through stimuli defined in the scenario model, such as by changing transportation conditions available to agents to move around the world. Finally, agents may affect the world such as when an agent emits pollutants into the modeled environment. Data about the population's beliefs and behaviors is integrated into variables held by individual agents. Distributions of the behaviors and beliefs of the agent population should be validated against the original survey data. The purpose of the ABM and framework is to ascertain the impact of the product and in order to do that the rules regarding the adoption of the product are paramount.

Choice models have been used previously in product development (Li and Azarm, 2000), social interactions (Brock and Durlauf, 2001), and ABM (He et al., 2014). An agent's choice of adoption can be modeled as a utility function as shown in Equation 1, where $U_{i}$ is the product utility as understood by agent $i$ and the utility is calculated for each of the $n$ agents. Agent $i$ will adopt the product if $U_{i}>0$. $U_{i}$ contains an observed portion represented by $V_{i}$ and an unobserved portion $\epsilon_{i}$. The observed portion includes the influence of all product, and TPB parameters where $j$ is the number of parameters as shown in Equation 2. $\beta$ weightings may vary for individual agents or may be constant for the entire population. Varying $\beta$ across the population shows different weightings of the factors. An example of this $\beta$ variance across the population would be that the preference varies by the age of the agent. The $S$ variables will vary based on conditions of the model and by agent interactions. By assuming a logit model, the unobserved portion of Equation 1 is assumed to follow a logistic distribution. The probability of agent $i$ adopting the product can be defined by Equation 3. Therefore as the model progresses and conditions change, the probability of an agent adopting the product can change.

$$
\begin{array}{lr}
U_{i}=V_{i}+\varepsilon_{i} & i \in\{1,2, \ldots, n\} \\
V_{i}=\beta_{1} S_{1}+\beta_{2} S_{2}+\cdots+\beta_{j} S_{j} & j \in\{1,2, \ldots, m\} \\
P_{i}=\frac{1}{1+e^{-V_{i}}} & i \in\{1,2, \ldots, n\}
\end{array}
$$

\subsection{Framework: Evaluation of Impact}

As agents adopt the product they will experience an impact. For example, if a product improves the health of an agent, that agent may be more likely to continue working, going to school, or survive. Measurements of aggregate impact across the population should be recorded for each run of the simulation. Depending on the model it may also be important to break down the impact by different demographic categories rather than a single measure of impact for the entire population. Product parameters can be iterated on in the model to see how changes in product parameters influence the impact of the product on society. It is important to note that because of the stochastic nature of an ABM, results will vary so it is important to run the model a sufficient number of times to understand the distribution of results. By continuing to iterate on the parameters a design team can understand what factors are most important to improve to maximize potential positive impact and minimize potential negative impact.

\section{FRAMEWORK ILLUSTRATION}

In order to demonstrate using the framework an illustration is presented in this section. During the COVID-19 pandemic the impact of face masks has been widely discussed. Because face masks are a product that most of the world has become familiar with in 2020, combined with the amount of data available surrounding COVID-19, it proved to be an excellent case for demonstrating the framework. 
Simplifications have been made to the model to enable comparisons. There is only one type of face mask on a given simulation run. This simplifies the model to a binary choice and makes the results clear as to which face mask has the greatest total impact. The model focuses on the United States as preferences may change in different countries and more data was available to construct the model based on communities in the United States.

\subsection{Illustration: Product Model}

Five mask designs with different attributes were evaluated in the model: (i) a control case with no masks, (ii) an N95 mask with high effectiveness but lower comfort, (iii) a cloth mask with medium effectiveness and comfort, (iv) a neck gaiter with low effectiveness but high comfort, and (v) an ideal mask that is very effective, comfortable, and aesthetic. Effectiveness was rated on a 0 to 5 scale, with 0 being no mask and 5 a mask that filters $100 \%$ of droplets. Comfort was rated on a -5 to 0 with 0 being no mask and all masks will be less comfortable than no mask. Aesthetics were rated on a -5 to 5 scale with 5 being a mask with excellent aesthetic qualities that will make a user want to wear it, while a score of -5 will deter use. Attributes of masks were built from data by Fischer et al. (2020). The attribute scores of each mask case can be seen in Table 1 .

Table 1. Mask attributes

\begin{tabular}{llll}
\hline Mask Type & Effectiveness & Comfort & Aesthetics \\
\hline None & 0 & 0 & N/A \\
N95 & 4.75 & -4.5 & -3 \\
Cloth & 2.5 & -2.5 & 3 \\
Neck Gaiter & 1 & -0.5 & 2 \\
Ideal Mask & 5 & 0 & 5 \\
\hline
\end{tabular}

\subsection{Illustration: Society Model}

The goal of the society model is to fully define the model population with demographics, preferences, movement patterns, and behaviors. Synthetic population attributes were based on Cape Elizabeth, Maine. The synthetic population was created using aggregate data from the 2019 US Census American Consumer Survey.

Assumptions and simplifications were made about the society to enable modeling such as all adults go to a workplace, and all children go to a school. The frequency of agents traveling to locations was defined with data from the American Time Use Survey (United States Bureau of Labor Statistics, 2020).

Relative user preferences were obtained through existing studies on mask use demographics (Igielnik, 2020) and perceptions (Howard, 2020). An assumption was made that older members of the population have a higher preference for mask efficacy due to higher mortality rates. The user preferences were used to obtain the utility function for person $i$ adopting the use of a face mask, see Equation 4 . Equation 4 is then substituted into Equation 3 to obtain the probability of agent $i$ adopting a mask on a given day in the model. The $\beta$ coefficients are the relative weighting of different factors by an individual and the $S_{i}$ terms are the scoring of that variable. Values and ranges for the variables of the utility function can be found in Table 2. The attitude toward mask scores, $S_{A T B}$, were initialized using a normal distribution with a median of -1 and standard deviation of 1 to simulate the population initially being more unfamiliar with face mask use (Igielnik, 2020). Social norm scores were set based on the percent of connections in the person's network that have adopted masks with a 5 being all connections have adopted masks and -5 being no connections adopting masks. Virus severity was defined by the percent of the population currently infected with the virus. These variables define utility of a face mask for each user.

$$
V_{n}=\beta_{A T B} S_{A T B}+\beta_{S N} S_{S N}+\beta_{P B C} S_{P B C}+\beta_{E} S_{E}+\beta_{C} S_{C}+\beta_{A} S_{A}+\beta_{V} S_{v}
$$

Individuals in the network built social connections based on a small world network structure (Watts and Strogatz, 1998) with their five closest neighbors and up to five individuals that share the same work place. It was asssumed that there was a ten percent chance of deleting a social connection and forming a new connection with a random individual in the population. 
Table 2. Variables and coefficients for DCA

\begin{tabular}{llllll}
\hline Variable & Weight & Weight Value & Score & Score Value/Range & Score Type \\
\hline Attitude toward behavior & $\beta_{A T B}$ & 0.4 & $S_{A T B}$ & $-5: 5$ & Dynamic \\
Social norms & $\beta_{S N}$ & 0.3 & $S_{S N}$ & $-5: 5$ & Dynamic \\
Perceived behavioral control & $\beta_{P B C}$ & 0.3 & $S_{P B C}$ & $0: 5$ & Static \\
Effectiveness & $\beta_{E}$ & $0.2,0.3,0.4^{*}$ & $S_{E}$ & $0: 5$ & Static \\
Comfort & $\beta_{C}$ & $0.4,0.3,0.2^{*}$ & $S_{C}$ & $-5: 0$ & Static \\
Aesthetics & $\beta_{A}$ & $0.2,0.15,0.1^{*}$ & $S_{A}$ & $-5: 5$ & Static \\
Virus Severity & $\beta_{V}$ & $0.2,0.3,0.4^{*}$ & $S_{V}$ & $-5: 5$ & Dynamic \\
\hline
\end{tabular}

*For weights with multiple values: 1 st value ages $19-39$, 2nd value ages $40-65$, and 3 rd value ages $65+$

\subsection{Illustration: Scenario Model}

The scenario model defines the scope and initial conditions of the simulation. The simulation was run for 5,376 time steps or about eight weeks. Each time step is equal to 15 minutes of real world time. Testing face mask impact during COVID-19 was the main goal of the scenario so 25 people were initially infected with the virus. Parameters of the virus such as mortality rates by age, infectiousness, and duration of illness were set according to data from the Centers for Disease Control and Prevention (COVID-19 Pandemic Planning Scenarios, 2020). In this model the scenario takes place in a closed system, so there are no new individuals to the population that may add cases of COVID-19. Parameters for mask mandates, the perceived behavioral control term in Equation 4, were included on a 0 to 5 scale with five being that masks are required by law, but to isolate the mask design impact from policy impacts, it was set at 1 for all scenarios which would be equivalent to suggesting the public wear a mask.

\subsection{Illustration: Impact Model}

The focus of Section 3 is to demonstrate use of the framework for social impact, but similar approaches could be taken with economic or environmental impact. Health and safety was selected as the impact category to assess from those presented by Rainock et al. (2018). To measure health and safety, indicators of the total number of COVID-19 cases and the number of deaths in the simulation were selected.

\subsection{Illustration: Agent-Based Model}

Most parameters of the ABM have been defined in the other input models (Sections 3.1-3.4). The ABM for this illustration was developed using the Netlogo software package (Wilensky, 1999). To define how the virus can spread within the model, interactions between individuals were defined as two agents occupying the same coordinate in space. If this occurred there was a probability based on the COVID-19 parameters that the non-infected individual would contract the virus from the infected individual. Once at a given location the individuals would move in a random walk for the duration they were in a location such as a workplace, school, or store to simulate random interactions that can take place throughout the day. By integrating the four input models in an ABM it is possible to model the health and safety impacts of face masks in the context of COVID-19.

\subsection{Illustration: Evaluation of Impact}

To understand the range of results 75 full simulations were completed for each potential mask. A summary of the results is presented in Table 3 . The results show that there is a statistically significant difference between all mask types and no masks with p-values $<0.0001$ from an analysis of variance test. A mask with low effectiveness, like a neck gaiter, still provides a significant reduction in COVID19 cases, but does not provide the same impact as a more effective mask. There was not a statistically significant difference in the number of COVID-19 cases between cloth, N95 and ideal masks with a p-value $>0.9$ from a Tukey-Kramer test.

In order to understand the individual mask attributes of effectiveness, comfort, and aesthetics, a sensitivity analysis was performed using the cloth mask type as the base. This mask type was selected because cloth masks have become ubiquitous during the pandemic. Each of the mask attributes was improved by one unit while the others were held constant. 
Table 3. Simulation results

\begin{tabular}{lrr|rr}
\hline & \multicolumn{2}{c}{ Total Cases } & \multicolumn{2}{c}{ Deaths } \\
\cline { 2 - 5 } Mask Type & \multicolumn{1}{c}{ Mean } & \multicolumn{1}{c}{$\sigma$} & Mean & \multicolumn{1}{l}{$\sigma$} \\
\hline No Mask & 2564.64 & 256.40 & 39.96 & 10.89 \\
Neck Gaiter & 408.36 & 208.38 & 6.64 & 4.08 \\
Cloth Mask & 63.00 & 14.28 & 2.12 & 1.29 \\
N95 Mask & 44.16 & 6.59 & 1.76 & 1.36 \\
Ideal Mask & 45.33 & 7.53 & 1.81 & 1.46 \\
\hline
\end{tabular}

Table 4. Sensitivity analysis

\begin{tabular}{llllllll}
\hline Mask & \multirow{2}{*}{ Effectiveness } & \multirow{2}{*}{ Aesthetics } & Comfort & $\begin{array}{l}\text { Mean } \\
\text { Total Cases }\end{array}$ & $\begin{array}{l}\sigma \\
\text { Total Cases }\end{array}$ & $\begin{array}{l}\text { Change in Cases } \\
\text { From Base }\end{array}$ & p-value \\
\hline Cloth Mask Base & 2.5 & 3 & -2.5 & 63.00 & 14.28 & N/A & N/A \\
Improved Effectiveness & 3.5 & 3 & -2.5 & 49.60 & 7.51 & -13.40 & $<0.0001$ \\
Improved Comfort & 2.5 & 3 & -1.5 & 64.27 & 14.24 & +1.27 & 0.93 \\
Improved Aesthetics & 2.5 & 4 & -2.5 & 63.87 & 13.70 & +0.87 & 0.98 \\
\hline
\end{tabular}

This sensitivity analysis shows that effectiveness is the most important attribute to improve by one unit to improve the positive impact. Effectiveness was the only attribute that provided a statistically significant difference. With the limited resources that a design team has the effectiveness attribute should be where the priority lies for improvement of the design.

This is how the framework would function in the design process. A model is built factoring in the product, society, particular scenario, and potential impacts. As the design team iterates on product inputs in the model they can see how the predicted impact of the product changes. By observing these changes over iterations of product parameters the design team can see opportunities to increase positive impact and reduce negative impact.

\subsection{Verification and Validation}

Efforts were made to validate the model. Although it is difficult to perform macro-validation of the entire model, micro-validation was carried out on the individual components of the model as suggested by Wilensky and Rand (2015). The synthetic populations were validated to have the same distributions as the real population, and virus parameters were validated to match real-world estimates (COVID-19 Pandemic Planning Scenarios, 2020). It was verified that mask parameters matched lab studies (Fischer et al., 2020) and user preferences (Howard, 2020). Qualitative measures of the results seem to match what would be expected based on different cases of face masks, but because the scenario cannot capture the complexities of the many different masks on the market and a high fidelity epidemiological model it is difficult to perform quantitative macro-validation.

\section{CONCLUSION}

By combining existing methods for impact assessment, and technology adoption into an ABM, the framework presented provides an outline for how a design team can quantitatively assess the potential impact of many design candidates before the product reaches the market, and identify opportunities of how the design can be changed to maximize positive impact. The framework presented allows impact to be measured for different population segments or for the entire target population. As the world continues to place greater importance on the impact of products, tools such as the framework presented in this paper will be a valuable asset for design teams.

\section{ACKNOWLEDGMENTS}

The authors acknowledge the support of the National Science Foundation, Grant CMMI-1761505. 


\section{REFERENCES}

Ajzen, I. (1991), 'The theory of planned behavior', Organizational Behavior and Human Decision Processes 50(2), 179-211. URL: https://doi.org/10.1016/0749-5978(91)90020-T

Axelrod, R. (1997), 'Advancing the art of simulation in the social sciences', Complexity 3(2), 16-22.

Benoît, C., Norris, G. A., Valdivia, S., Ciroth, A., Moberg, A., Bos, U., Prakash, S., Ugaya, C. and Beck, T. (2010), 'The guidelines for social life cycle assessment of products: just in time!', The International Journal of Life Cycle Assessment 15(2), 156-163. URL: https://doi.org/10.1007/s11367-009-0147-8

Bonabeau, E. (2002), 'Agent-based modeling: Methods and techniques for simulating human systems', Proceedings of the National Academy of Sciences of the United States of America 99(SUPPL. 3), 7280-7287. URL: https://doi.org/10.1073/pnas.082080899

Brock, W. A. and Durlauf, S. N. (2001), 'Discrete choice with social interactions', Review of Economic Studies. URL: https://doi.org/10.1111/1467-937X.00168

Burdge, R. J. (2015), A community guide to social impact assessment, fourth edn, Social Ecology Press, Huntsville.

Chen, X. and Kelly, T. F. (2015), 'B-Corps-A Growing Form of Social Enterprise: Tracing Their Progress and Assessing Their Performance', Journal of Leadership \& Organizational Studies 22(1), 102-114. URL: https://doi.org/10.1177/1548051814532529

COVID-19 Pandemic Planning Scenarios (2020). URL: https://www.cdc.gov/coronavirus/2019ncov/hcp/planning-scenarios.html

Esteves, A. M., Franks, D. and Vanclay, F. (2012), 'Social impact assessment: the state of the art', Impact Assessment and Project Appraisal 30(1), 34 42. URL: https://doi.org/10.1080/14615517.2012.660356

Fischer, E. P., Fischer, M. C., Grass, D., Henrion, I., Warren, W. S. and Westman, E. (2020), 'Low-cost measurement of face mask efficacy for filtering expelled droplets during speech', Science Advances 6(36). URL: https://doi.org/10.1126/sciadv.abd3083

Fontes, J., Gaasbeek, A., Goedkoop, M., Contreras, S. and Evitts, S. (2016), 'Handbook for Product Social Impact Assessment 3.0'. URL: https://doi.org/10.13140/RG.2.2.23821.74720

Fontes, J., Tarne, P., Traverso, M. and Bernstein, P. (2018), 'Product social impact assessment', The International Journal of Life Cycle Assessment 23(3), 547-555. URL: https://doi.org/10.1007/s11367-016-1125-6

Gargiulo, F., Ternes, S., Huet, S. and Deffuant, G. (2010), 'An iterative approach for generating statistically realistic populations of households', PloS one 5(1), e8828. URL: https://doi.org/10.1371/journal.pone.0008828

Gelles, D. and Yaffe-Bellany, D. (2019), 'Shareholder Value Is No Longer Everything, Top C.E.O.s Say'. URL: https://www.nytimes.com/2019/08/19/business/business-roundtable-ceoscorporations. html

He, L., Wang, M., Chen, W. and Conzelmann, G. (2014), 'Incorporating social impact on new product adoption in choice modeling: A case study in green vehicles', Transportation Research Part D: Transport and Environment 32, 421-434. URL: http://dx.doi.org/10.1016/j.trd.2014.08.007

Howard, M. C. (2020), 'Understanding face mask use to prevent coronavirus and other illnesses: Development of a multidimensional face mask perceptions scale', British Journal of Health Psychology 25(4), 912-924.

URL: https://doi.org/10.1111/bjhp.12453

Igielnik, R. (2020), Most Americans say they regularly wore a mask in stores in the past month; fewer see others doing it, Technical report, Pew Research Center. URL:

https://www.pewresearch.org/fact-tank/2020/06/23/most-americans-say-they-regularly-wore-amaskin-stores-in-the-past-month-fewer-see-others-doing-it/

Latora, V. and Marchiori, M. (2001), 'Efficient Behavior of Small-World Networks', Phys. Rev. Lett. 87(19), 198701. URL: https://doi.org/10.1103/PhysRevLett.87.198701

Li, H. and Azarm, S. (2000), 'Product design selection under uncertainty and with competitive advantage', Journal of Mechanical Design, Transactions of the ASME. URL: https://doi.org/10.1115/1.1311788

Macal, C. M. and North, M. J. (2005), 'Tutorial on agent-based modeling and simulation', Proceedings - Winter Simulation Conference 2005, 2-15.

Mattson, C. A. and Winter, A. G. (2016), 'Why the Developing World Needs Mechanical Design', Journal of Mechanical Design 138(7). URL: https://doi.org/10.1115/1.4033549

Müller, K. and Axhausen, K. W. (2010), 'Population synthesis for microsimulation: State of the art', Arbeitsberichte Verkehrs-und Raumplanung 638.

Norman, W. and MacDonald, C. (2004), 'Getting to the Bottom of "Triple Bottom Line"', Business Ethics Quarterly. URL: https://doi.org/10.5840/beq200414211

Pack, A. T., Rose Phipps, E., Mattson, C. A. and Dahlin, E. C. (2019), 'Social Impact in Product Design, An Exploration of Current Industry Practices', Journal of Mechanical Design 142(7). URL: https://doi.org/10.1115/1.4045448 
Pakravan, M. and MacCarty, N. (2020), 'an Agent-Based Model for Adoption of Clean Technology Using the Theory of Planned Behavior', Journal of Mechanical Design pp. 1-19. URL: https://doi.org/10.1115/1.4047901

Pavlou, P. A. and Fygenson, M. (2006), 'Understanding and Predicting Electronic Commerce Adoption: An Extension of the Theory of Planned Behavior', MIS Quarterly 30(1), 115-143. URL: https://doi.org/10.2307/25148720

Rai, V. and Robinson, S. A. (2015), 'Agent-based modeling of energy technology adoption: Empirical integration of social, behavioral, economic, and environmental factors', Environmental Modelling \& Software 70, 163-177. URL: https://doi.org/10.1016/j.envsoft.2015.04.014

Rainock, M., Everett, D., Pack, A., Dahlin, E. C. and Mattson, C. A. (2018), 'The social impacts of products: a review', Impact Assessment and Project Appraisal. URL: https://doi.org/10.1080/14615517.2018.1445176

Rogers, E. M. (1995), Diffusion of Innovations, Fourth Edition, fourth edn, Free Press, New York.

Schelling, T. C. (1971), 'Dynamic models of segregation', The Journal of Mathematical Sociology 1(2), 143-186. URL: https://10.0.4.56/0022250X.1971.9989794

Schwarz, N. and Ernst, A. (2012), 'Agent-based modeling of the diffusion of environmental innovations - An empirical approach', International Journal of Agent Technologies and Systems 4, 497-511. URL: https://doi.org/10.1016/j.techfore.2008.03.024

Smaldino, P. E., Pickett, C. L., Sherman, J. and Schank, J. (2012), 'An Agent-Based Model of Social Identity Dynamics', Journal of Artificial Societies and Social Simulation 15(4), 1-17. URL: https://doi.org/10.18564/jasss.2030

Squazzoni, F. (2010), 'The Impact of Agent-Based Models in the Social Sciences After 15 Years of Incursions'. URL: https://doi.org/10.2307/23723517

Stevenson, P. D., Mattson, C. A. and Dahlin, E. C. (2020), 'A Method for Creating Product Social Impact Models of Engineered Products', Journal of Mechanical Design, Transactions of the ASME. URL: https://doi.org/10.1115/1.4044161

United States Bureau of Labor Statistics (2020), 'American Time Use Survey (ATUS): Arts Activities, [United States], 2003-2018'.

Venkatesh, V., Morris, M. G., Davis, G. B. and Davis, F. D. (2003), 'User acceptance of information technology: Toward a unified view', MIS Quarterly: Management Information Systems. URL: https://doi.org/10.2307/30036540

Vicsek, T. (2002), 'Complexity: The bigger picture', Nature 418(6894), 131. URL: https://doi.org/10.1038/418131a

Wassenaar, H. J. and Chen, W. (2003), 'An Approach to Decision-Based Design With Discrete Choice Analysis for Demand Modeling ', Journal of Mechanical Design 125(3), 490-497. URL: https://doi.org/10.1115/1.1587156

Watts, D. J. and Strogatz, S. H. (1998), 'Collective dynamics of 'small-world' networks', Nature 393(6684), 440-442. URL: https://doi.org/10.1038/30918

Wilensky, U. (1999), 'NetLogo. http://ccl.northwestern.edu/netlogo/.', Center for Connected Learning and ComputerBased Modeling Northwestern University Evanston IL.

Wilensky, U. and Rand, W. (2015), Verification, Validation, and Replication, in 'An Introduction to Agent-based Modeling', The MIT Press, Cambridge, pp. 325-336.

Xu, Z., Glass, K., Lau, C. L., Geard, N., Graves, P. and Clements, A. (2017), 'A synthetic population for modelling the dynamics of infectious disease transmission in American Samoa', Scientific Reports 7(1), 1-9. URL: https://doi.org/10.1038/s41598-017-17093-8 